\title{
The Effect of Spinal Instrumentation on Lumbar Intradiscal Pressure
}

\author{
Eiji Abe, Troy Nickel, ${ }^{1}$ Glenn R. Buttermann, ${ }^{1}$ \\ $J_{A C K}$ L. Lewis ${ }^{1}$ and Ensor E. Transfeldt ${ }^{1}$ \\ Department of Orthopaedic Surgery, Akita University \\ School of Medicine, Akita 010-8543, and ${ }^{1}$ Bioengineering \\ Laboratory at the Department of Orthopedic Surgery, \\ University of Minnesota, Minneapolis, MN 55455, USA
}

\begin{abstract}
Abe, E., Nickel, T., Buttermann, G.R., Lewis, J.L. and Transfeldt, E.E. The Effect of Spinal Instrumentation on Lumbar Intradiscal Pressure. Tohoku J. Exp. Med., 1999, 187 (3), 237-247 — The purpose of this study was to investigate the effect of spinal instrumentation on the intradiscal pressure (IDP) within the fixed motion segment. In vitro biomechanical testing was performed in six single functional spinal units of fresh calf lumbar spines using a pressure needle transducer. Various loads were applied by a materials testing system device. In addition to intact spine (control), anterior spinal instrumentation (ASI) and pedicle screw fixation (PS) constructs, as well as destabilized spine were tested. Relative to the control, the destabilized spine tended to have an increased IDP; by $15 \%$ in axial compression and by $9-36 \%$ in flexion-extension. Compared to the control, PS decreased the IDP by $23 \%$ in axial loading and $51 \%$ in extension loading and increased it by $60 \%$ in flexion for each loading. ASI decreased the IDP by $32 \%$ in flexion and $1 \%$ in extension. Lateral bending produced symmetrical changes of IDP in the control and destabilized spine, but no change in the PS construct. The IDP of the ASI construct was decreased by $77 \%$ in ipsilateral bending and increased by $22 \%$ in contralateral bending. These results demonstrated that eccentric loading from the spinal instruments increased IDP and significant disc pressure may still exist despite an increase in motion segment stiffness after lumbar stabilization. ____ intradiscal pressure; load sharing; pedicle screw fixation; anterior spinal instrumentation (C) 1999 Tohoku University Medical Press
\end{abstract}

Various spinal instruments have been employed for spinal trauma or disorders and there are many reports of instrumentation failure, especially breakage of pedicle screws (Matsuzaki et al. 1990; Dickman et al. 1992; Esses et al. 1993; McLain et al. 1993; Niu et al. 1996; Stovall et al. 1996). Although there have been several biomechanical studies done on the instrumented spine, most of them

Received February 12, 1999; revision accepted for publication Feburary 26, 1999. Address for reprints: Eiji Abe, Department of Orthopedic Surgery, Akita University School of Medicine, 1-1-1 Hondo, Akita 010-8543, Japan.

e-mail: eijiabe@med.akita-u.ac.jp 
concentrated on the stiffness of the spine. Little is known about load sharing of the intervertebral disc or the simulated anterior strut graft within the fixed segment with or without instrumentation. Load sharing of the spine with spinal instrumentation is usually measured with strain gauges installed into the spinal instruments or simulated vertebral body grafts (Chiba et al. 1996; Harris et al. 1996; Rohlmann et al. 1996; Kanayama et al. 1997). Nachemson and Morris (1964) clarified total load to the intervertebral disc by measuring the intradiscal pressure (IDP); therefore, we think the IDP within the fixed segment with the spinal instrument also correlates directly with the total load to the disc and closely with the load sharing of the spinal instrument. There have been few studies done on the effect of spinal instrumentation or spinal fusion on the IDP within the fixed segment with widely varying and sometimes controversial results (Nachemson and Morris 1964; Rolander 1966; Weinhoffer et al. 1995; Cunningham et al. 1997). But to our knowledge, there has been no biomechanical study about either the kind of loading which should be restricted to prevent instrumentation failure after surgery or the effect of anterior spinal instrumentation on the IDP within the fixed segment. The purpose of the present study is to evaluate the effect of anterior and posterior spinal instrumentation on loading stress to the disc by means of measuring the IDP within the fixed segment of various loadings.

\section{Materials and Methods}

\section{Preparation of materials}

Fresh-frozen calf lumbar spines harvested from six calves (80 to $120 \mathrm{~kg}$ body weight) were used in this investigation. The specimens were thawed to room temperature for 12 hours and one functional spinal units of the L4-L5 segment were isolated. Each isolated segment was immediately frozen at $-20^{\circ} \mathrm{C}$ in double thickness plastic bags. Just before the preparation and mechanical testing, the isolated segments were thawed to room temperature for about 12 hours. The surrounding soft tissue and muscles were dissected off the segments, with care being taken to preserve the ligamentous structures, facet joint capsules and discs. The upper and lower vertebrae of each isolated segment were anchored with 4 to 6 stainless steel screws and embedded in metal fixtures using bone cement (methylmethacrylate). Great care was taken to place the potted vertebrae in a parallel and coaxial direction to fit into jigs, which were attached to a loading device in a materials testing system (MTS) machine and encompassed a system of six linear variable different transformers (LVDT) for following motion measurements (Panjabi et al. 1981). The specimens were kept moist with saline spray during the preparation of the specimen and the mechanical testing. The intervertebral disc sizes of the L4-L5 specimens were $41.8 \pm 4.4 \mathrm{~mm}$ in antero-posterior diameter and $48.8 \pm 3.5 \mathrm{~mm}$ in lateral diameter. 


\section{Spinal constructs}

The following four different lumbar constructs were tested in six L4-L5 segments with $300 \mathrm{~N}$ loading: 1) the intact spine (control), 2) pedicle screw fixation (PS) construct, 3) anterior spinal instrumentation (ASI) construct and 4) destabilized spine. PS was done in situ with the Texas Scottish Rite Hospital (TSRH) spinal system (Sofamor Daneck, Memphis, TN, USA), which consists of 4 stainless-steel variable angle screws $(6.5 \mathrm{~mm}$ in diameter and $40 \mathrm{~mm}$ in threaded length) and 2 rods (6.35 mm in diameter) (Fig. 2a). ASI was done in situ with the Kaneda device (Mizuho Ikakogyo Co., Tokyo), which consists of 4 stainlesssteel vertebral screws $(5.5 \mathrm{~mm}$ in diameter and $40 \mathrm{~mm}$ in threaded length) and 2 threaded rods (4.5 mm in outer diameter) (Fig. 2b). All of the vertebral screws penetrated the contra lateral cortex of the vertebral bodies. The destabilized spines were created by bilateral complete facetectomy and transection of the ligamentous structures in the posterior column.

\section{Material loading system}

The biomechanical tests were performed using a servohydraulic materials testing machine (MTS; 858 Bionix Testing System, Minneapolis, MN, USA). An axial compression force of 0 to $300 \mathrm{~N}$ was applied on the loading board secured to

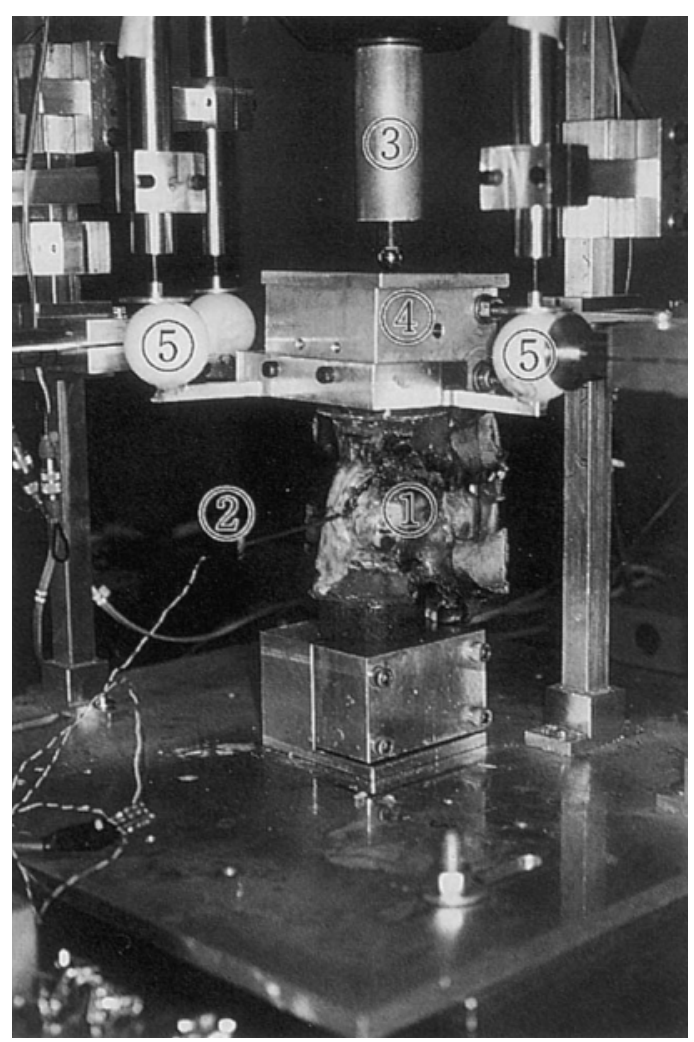

Fig. 1. Intradiscal pressure measurement system.

1, a specimen (calf lumbar spine); 2, needle-type microtransducer inserted into the intervertebral disc; 3, load cell (MTS machine); 4, loading plate; 5, six linear variable different transformers (LVDT). 
the upper end of a specimen with a $10 \mathrm{~mm}$ diameter iron ball at a loading rate of $60 \mathrm{~N} /$ seconds (Fig. 1). Before the loading tests, each specimen was preconditioned with axial compression $(0-300 \mathrm{~N})$ four or five times until stabilization under monitoring of IDP was observed. Each test condition was replicated three times to ensure repeatability.

The $0-300 \mathrm{~N}$ loading tests of six L4-L5 segments were first done with the intact spine, then with PS construct, ASI construct, and finally the destabilized spine. Testing modes included axial compression to the axis of rotation of the intact spine, flexion-extension and lateral bending. The order of the tests was not randomized because of the violation of the facet joint and ligamentum flavum.

The axis of rotation of the specimen was determined by LVDT output monitoring on the intact spine of each specimen. The loading point of axial compression is at the axis of rotation of the control; those of flexion, extension and lateral bending were at $10 \mathrm{~mm}, 20 \mathrm{~mm}$ and $30 \mathrm{~mm}$ anterior, posterior and lateral to the axis of rotation in each intact spine, respectively. The terms Flex 1, 2 and 3 indicate $300 \mathrm{~N}$ loading at 10,20 and $30 \mathrm{~mm}$ anterior to the axis of rotation of the intact spine, i.e., 3,6 and $9 \mathrm{Nm}$ at $300 \mathrm{~N}$ loading anteriorly. Ext 1,2,3 and Lateral bending 1,2,3 indicate 3,6 and $9 \mathrm{Nm}$ in $300 \mathrm{~N}$ loading posteriorly and laterally, respectively.

\section{Intradiscal pressure measurement}

IDP was measured directly by a specially designed needle-type micropressure transducer (Model 3521-500 with an outer diameter of $2.11 \mathrm{~mm}$, Robert A. Denton Co., Rochester Hill, MI, USA). The pressure transducer voltage output is linearly related to pressure for values up to $3.4 \mathrm{MPa}$ for 0 to $1000 \mathrm{~N}$ loading. The transducer was placed into the center of the disc through the anterior portion of the annulus and attached with sutures and super-glue to maintain it stationary position between tests. The position of the tip of the transducer was confirmed by 2-directional $\mathrm{x}$-ray before the measurements. Pressure data were recorded by computer data acquisition. IDP change (net IDP) was defined as the difference between the maximum IDP at loading and the lowest IDP before loading. IDP changes at each moment in each spinal construct were an average value of three median measured values.

\section{Motion monitoring}

The motion of the specimen was measured with a six-degree-of-freedom LVDT system. This tester describes the motion of the segment in the form of three rotations, i.e., axial rotation, flexion/extension bending, and lateral bending, and three translations, i.e., anterior/posterior shear, axial extension/compression, and lateral shear. The system's measurement errors are $0.4 \%$ for rotation, and $0.9 \%$ for translation (Panjabi et al. 1981). These data were recorded simultaneously with the pressure data by computer data acquisition. 
a

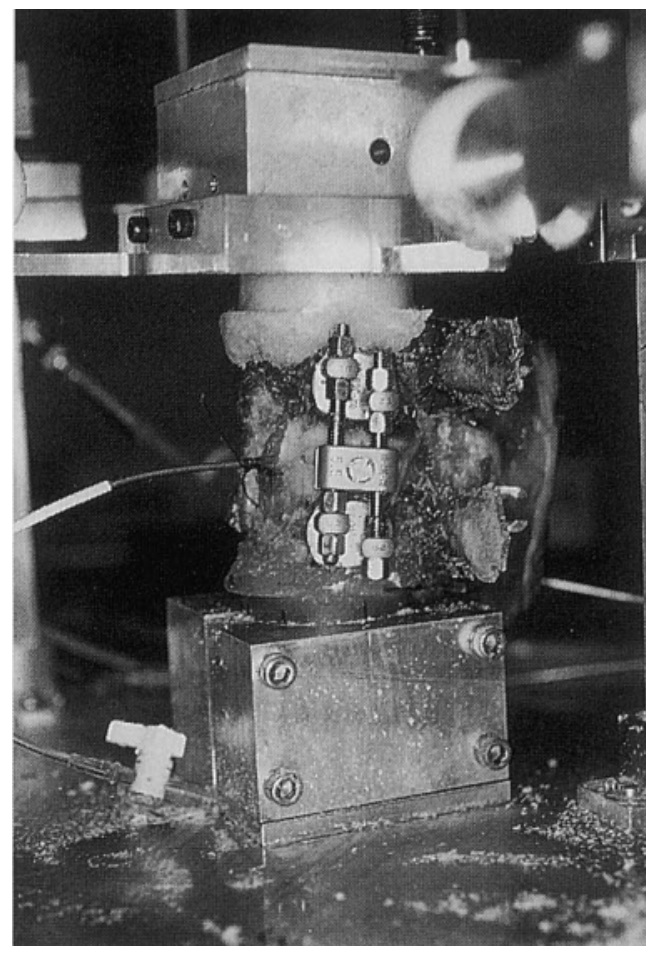

b

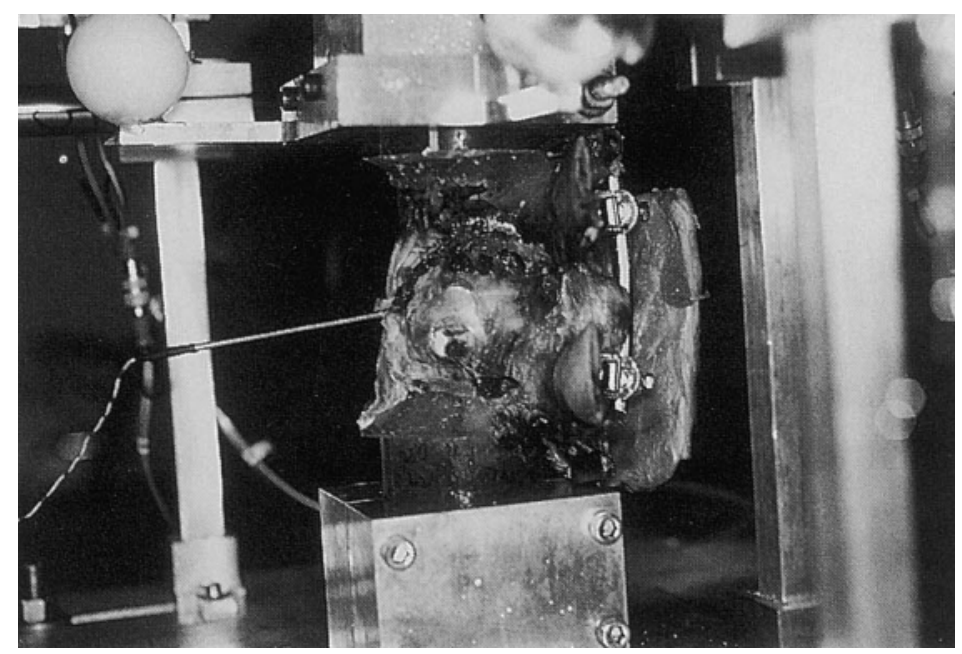

Fig. 2. a, Lateral view of the spine with pedicle screw fixation (TSRH system); b, Lateral view of the spine with anterior spinal instrumention (Kaneda device).

\section{Statistical analysis}

The statistical analysis of the biomechanical data included descriptive statistics, repeated-measures analysis of variance, and the post hoc Student-NewmanKeuls method for multiple comparisons between groups (Fisher's protected least significant difference). Probability values less than 5\% were considered significant.

\section{Results}

\section{Nonloading condition}

The IDP values in the nonloading condition among the spines with the same 
instrumentation were changed easily by the spinal instrument assembly technique. In order to decrease the effect of the instrument assembly technique as much as possible, we used instruments easily to adjust to the spine in situ. The IDP in this condition was $78.3 \pm 13.9 \mathrm{kPa}$ (mean \pm S.D.) in the control spine, $52.7 \pm$ $5.7 \mathrm{kPa}$ in the destabilized spine, $102.5 \pm 24.8 \mathrm{kPa}$ in the $\mathrm{PS}$, and $126.7 \pm 18.8 \mathrm{kPa}$ in the ASI. Although there were wide variations even in the IDP values for the same instruments, there were significant increases in the IDP of the ASI and PS construct, compared to the control (ASI: $p<0.0001$, PS: $p<0.05$ ). The IDP of the destabilized spine was decreased compared to the control $(p<0.05)$.

\section{Axial compression}

The IDP showed a quite linear response to the applied axial load in the different spinal constructs (Fig. 3). The IDP of the destabilized spine was increased by $15 \%$ compared to the control under $300 \mathrm{~N}$ loading, but this difference was not significant $(p=0.068)$. The IDP value of the ASI construct was decreased by $9 \%$ and that of the PS construct by $9-23 \%$, but significance was limited to the PS construct $(p<0.01)$. Compared to the destabilized spine, the ASI decreased the disc pressure by $21 \%$ and the PS decreased it by $33 \%$ (ASI: $p<0.01$, PS: $p<0.001$ ) (Fig. 4).

\section{Flexion and extension}

From Ext. 3 to Flex. 3, the IDP values of the control spine and the destabilized spine changed simultaneously and almost in parallel in proportion to the size

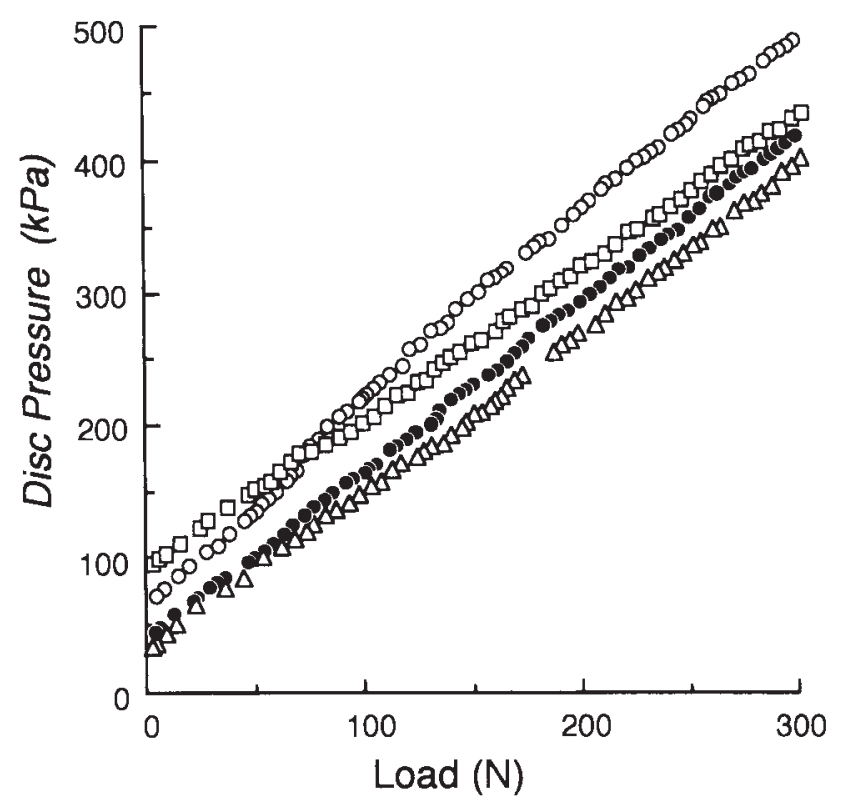

Fig. 3. Load-intradiscal pressure curve among the different instruments. ASI: anterior spinal instrumentation; PS: pedicle screw fixation; Destabilized: destabilized posterior column; Control: intact spine. -, Control; $\triangle$, Destabilized; $\bigcirc$, ASI; $\square$, PS. 


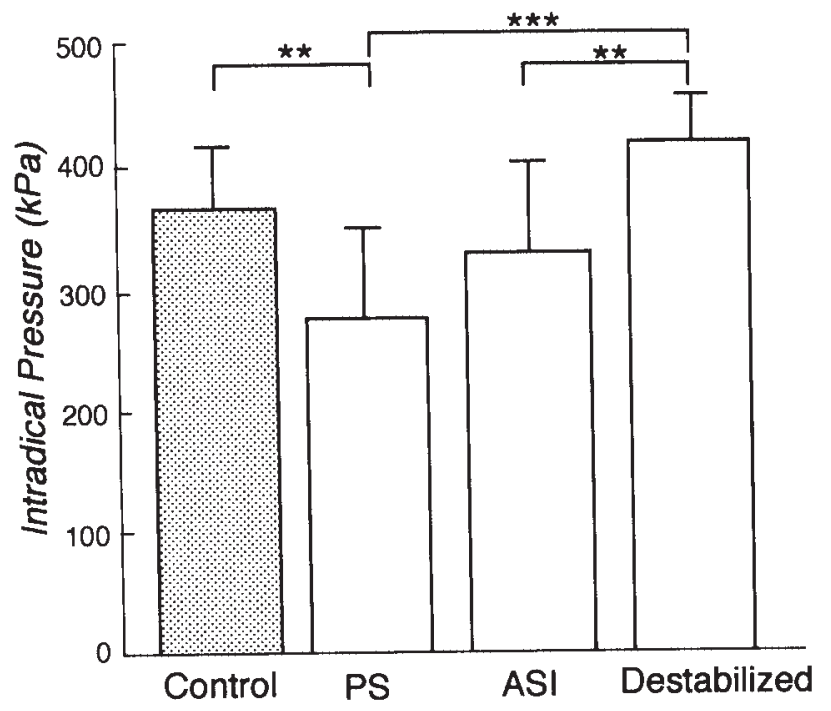

Fig. 4. Lumbar intradiscal pressure under axial compression loading in response to the intact spine (Control), pedicle screw fixation (PS), anterior spinal instrumentation (ASI), and spinal destabilization (Destabilized) under $300 \mathrm{~N}$ loading. The data are mean \pm s.e. values from six specimens. The error bar signifies S.E., and ${ }^{* *} p<0.01,{ }^{* * *} p<0.001$.

of each moment, but the lowest pressure of the control was at the axis of rotation, while that of the destabilized spine shifted to the anterior side at Flex. 1 ( $3 \mathrm{Nm}$ : $300 \mathrm{~N}$ loading at a point $10 \mathrm{~mm}$ anterior to the axis of rotation of the control spine). The IDP of the destabilized spine increased by $9-36 \%$ compared to the control in each bending moment in flexion-extension. The IDP of the PS increased almost linearly depending on the magnitude of the moment from Ext. 3 $(-9 \mathrm{Nm})$ to Flex. $3(9 \mathrm{Nm})$ and it was increased by $60 \%$ in Flex. 3 and decreased by $51 \%$ in Ext. 3 compared to axial compression. It was decreased by $5 \%$ in Flex. 3 and $71 \%$ in Ext. 3 compared to the control in each moment, and increased by $232 \%$ in Flex. 3 compared to the lowest extension values. The change in IDP values of the PS construct was significant only in extension compared to the control (Ext. 2 and 3, $p<0.0001$; Ext. 1, $p<0.001$ ). By contrast, the IDP of the ASI construct decreased constantly from Ext. 3 to Flex. 3. The IDP value of the ASI construct was almost the same as the control values and decreased by $32 \%$ in Flex. 3 (Fig. 5).

\section{Lateral bending}

Lateral bending produced symmetrical changes of IDP in the control and the destabilized constructs and their IDP was increased in proportion to the size of each moment, however, there was no change in the IDP of the PS construct compared with the axial loading to the axis of rotation. The IDP of the ASI construct was significantly decreased for ipsilateral bending (loading closer to the instrument), but increased for contralateral bending (loading farther from the instrument). Its IDP increased by $22 \%$ in RB 3 (contralateral bending) and decreased by $77 \%$ in LB 3 (ipsilateral bending). It decreased by $412 \%$ almost 


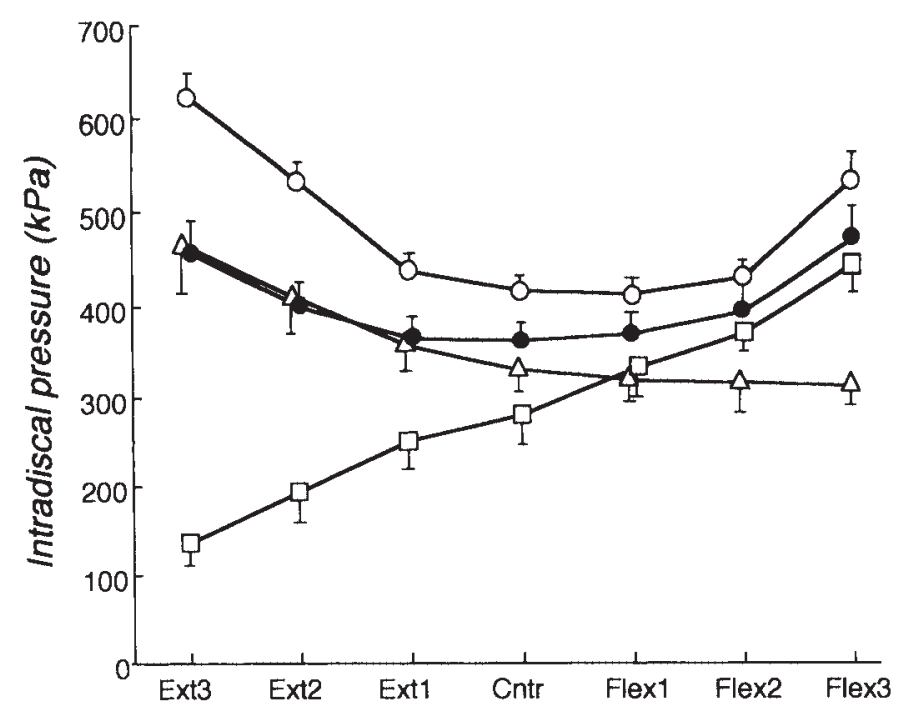

Fig. 5. Intradiscal pressure changes in flexion-extension with different stabilization and moment conditions under $300 \mathrm{~N}$ loading. The data are mean \pm s.E. values from six specimens. Ext. 3: $300 \mathrm{~N}$ loading at $30 \mathrm{~mm}$ posterior to the center of motion, i.e., $-9 \mathrm{Nm}$. Flex. 3: $300 \mathrm{~N}$ loading at $30 \mathrm{~mm}$ anterior to the center of motion, i.e., $+9 \mathrm{Nm}$. Y-axis: intradiscal pressure.

-, control (intact spine); $\bigcirc$, destabilized spine; 1., pedicle screw fixation; $\triangle$, anterior spinal instrumentation.

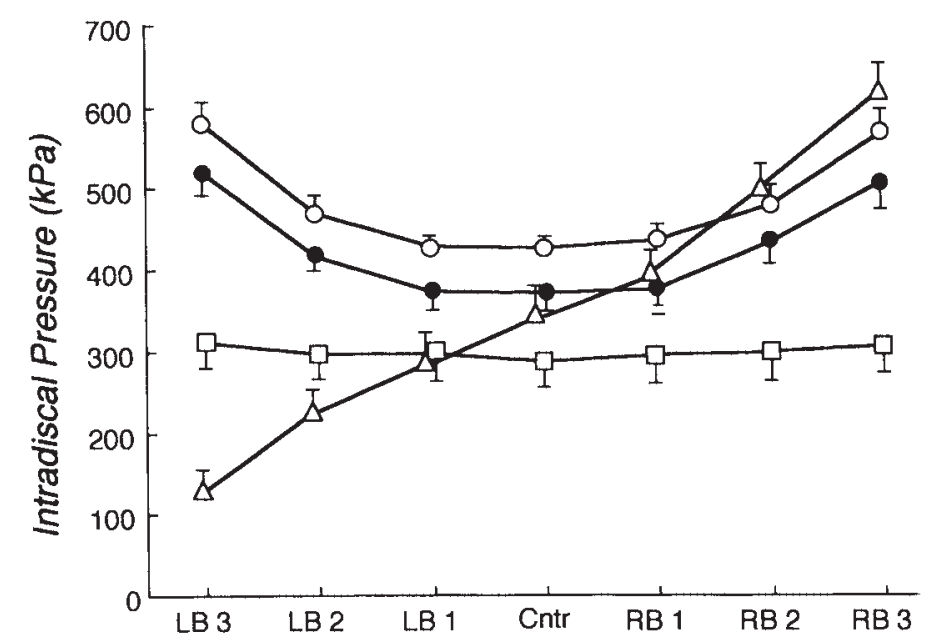

Fig. 6. Intradiscal pressure changes with lateral bending in different stabilization and moment conditions. The data are mean \pm s.e. values from six specimens. LB 3: $300 \mathrm{~N}$ loading at a point $30 \mathrm{~mm}$ left lateral to the center of motion. $y$-axis: intradiscal pressure.

-, control (intact spine); $O$, destabilized spine; $\ldots$, pedicle screw fixation; $\triangle$, anterior spinal instrumentation.

linearly from the loading farthest from the instrument to the loading closest to the instrument (Fig. 6).

\section{Discussion}

The present study was designed to evaluate the effects of spinal destabilization and anterior and posterior spinal instrumentation on IDP. Although we 
used 8-week-old calf spines because they have no degeneration and are similar size as adult human lumbar spines (Cotterill et al. 1986), the calf lumbar spine has slight kyphosis ( 0 to 5 degree), different contours of the facet joints, a small range of motions, six lumbar vertebrae and longer transverse processes. The first three points, especially the kyphotic curvature, may have affected the results of the present biomechanical testing.

Under axial compression, the IDP increased by $13 \%$ in the destabilized spine compared to the control in the present calf lumbar spine study $(p=0.068)$. In a human cadaver spine study, the IDP was increased significantly by $15-24 \%$ after posterior column disruption (Nachemson 1960; Cunningham et al. 1997). The difference between their findings and ours may relate to kyphotic and lordotic spine. The load sharing of the posterior column in a lordotic spine may be larger than that in a kyphotic spine just as extension increased the load sharing of the posterior column (Adamus et al. 1994). Another reason might be that our mechanical testing was done with monosegments, whereas their tests were done with multi-segmental spines.

The reduction in the IDP under PS was $23 \%$ in axial compression in the present study $(p<0.01)$. Its values was $55 \%$ in PS (Cunningham et al. 1997), $50 \%$ in simulated posterolateral fusion (Rolander 1966) in a human cadaver study, and $30 \%$ in posterior fusion in an in vivo study (Nachemson and Morris 1964). Compared to these reports, our data showed a somewhat small reduction of the IDP in the PS constructs. These differences also may well be due to the monosegmental vs. multisegmental and the kyphotic vs. lordotic spine difference.

In flexion, extension and lateral bending, the IDP of the control and destabilized spines increased similarly in proportion to the magnitude of the moment. This increase of IDP in the control is consistent with the findings of Rolander (1966), Nachemson and Elfström (1970) and Adams et al. (1994). The IDP values of the destabilized spine were always $9-36 \%$ higher than the control value during dynamic loading. The IDP of the PS construct was little changed by lateral bending. However, it increased linearly depending on the magnitude of the moment from extension to flexion, and was increased by $60 \%$ in flexion and decreased by $51 \%$ in extension compared to axial compression. The IDP in the PS construct reached a value as high as for the control spine at flexion. This result is partially consistent with that of Weinhoffer et al. (1995). They reported the IDP within the fixed segment with PS increased in flexion more than the IDP in the non-instrumented spine. But the results of Cunningham et al. (1997) differ from our results in this point. In their study, the IDP in flexion was still significantly lower than the IDP of the intact spine. This discrepancy seems to arise from the magnitude of flexion angle: Their angle was small in spite that it was a multisegmental spine study, therefore, flexion moment was so small that the loading was almost equal to axial loading and indeed his results nearly correspond to ours in Flexion $1(3 \mathrm{Nm})$ of small flexion moment. 
By contrast, the IDP of the ASI construct increased markedly with lateral bending to the opposite side of the instrument and decreased with bending to the same side. It increased linearly depending on the magnitude of the moment from the instrument and became higher than the control value in contralateral loading $(p<0.001)$. The IDP of the ASI construct at flexion and extension showed reverse changes compared with the PS construct; the IDP decreased in flexion and increased in extension.

The current study clearly demonstrated significantly high IDP in the disc at flexion in the PS construct and with contralateral bending in the ASI construct. A high IDP means heavy mechanical loading not only to the disc but also to the instruments themselves such as the rigid pedicle screw systems or the rigid anterior spinal instruments which are stiffer than single motion segments of the intact spine. Heavy mechanical loading on the disc or the instrument within the fixed segment seems to be one of the factors causing discogenic lower back pain (Wetherley et al. 1986; Kawakami et al. 1997; Suk et al. 1997) or instrumentation failure after posterolateral fusion or pedicle screw fixation. If there is no anterior support with anterior strut bone graft or vertebral spacers in the pedicle screw fixation, the motion of the anterior flexion should be restricted in order to prevent instrumentation failure. Similarly, loading or lateral bending to the contralateral side of the anterior spinal instrument or extension should be restricted until solid fusion is achieved.

\section{References}

1) Adams, M.A., McNally, D.S., Chinn, H. \& Dolan, P. (1994) Posture and the compressive strength of the lumbar spine. Clin. Biomech., 9, 5-14.

2) Chiba, M., McLain, R.F., Yerby, S.A., Moseley, T.A., Smith, T.S. \& Benson, D.R. (1996) Short-segment pedicle instrumentation: Biomechanical analysis of supplemental hook fixation. Spine, 21, 288-294.

3) Cotterill, P.C., Kostuik, J.P., D’Angelo, G., Fernie, G.R. \& Maki, B.E. (1986) An anatomical comparison of the human and bovine thoracolumbar spine. J. Orthop. Res., 4, 298-303.

4) Cunningham, B.W., Kotani, Y., McNulty, P.C., Cappuccino, A. \& McAfee, P.C. (1997) The effect of spinal destablization and instrumentation on lumbar intradiscal pressure: An in vitro biomechanical analysis. Spine, 22, 2655-2663.

5) Dickman, C.A., Fessler, R.G., MacMillan, M. \& Haid, R.W. (1992) Transpedicular screw-rod fixation of the lumbar spine: Operative technique and outcome in 104 cases. J. Neurosurg., 77, 860-870.

6) Esses, S.I., Sachs, B.L. \& Dreyzin, V. (1993) Complications associated with the technique of pedicle screw fixation: A selected survey of ABS members. Spine, 18, 2231-2238.

7) Harris, M.B., Thomas, K.A., Igram, C.M. \& Bearden, C.M. (1996) The effect of anterior thoracolumbar plate application on the compressive loading of the strut graft. Spine, 21, 1487-1493.

8) Kanayama, M., Cunningham, B.W., Weis, J.C., Parker, L.M., Kaneda, K. \& McAfee, P.C. (1997) Maturation of the posterolateral spinal fusion and its effect on loadsharing of spinal instrumentation: An in vivo sheep model. J. Bone Joint Surg. 
(Am.), 79, 1710-1720.

9) Kawakami, M., Tamaki, T., Yoshida, M., Ando, M. \& Hayashi, N. (1997) Lumbar complaints after posterolateral fusion in the lumbar spine. Spine Spinal Cord, 10, 599-604. (in Japanese with English abstract)

10) Matsuzaki, H., Tokuhashi, Y., Matsumoto, F., Hoshino, M., Kiuchi, T. \& Toriyama, S. (1990) Problems and solutions of pedicle screw plate fixation of lumbar spine. Spine, 15, 1159-1165.

11) McLain, R.F., Sparting, E. \& Benson, D.R. (1993) Early failure of short-segment pedicle instrumentation for thoracolumbar fractures. A preliminary report. J. Bone Joint Surg. (Am.), 75, 162-167.

12) Nachemson, A. (1960) Lumbar intradiscal pressure. Acta Orthop. Scand. (Suppl.), 43, 1-104.

13) Nachemson, A. \& Morris, C.J. (1964) In vivo measurements of intradiscal pressure. J. Bone Joint Surg. (Am.), 46, 1077-1092.

14) Nachemson, A. \& Elfström, G. (1970) Intravital dynamic pressure measurements in lumbar discs: A study of common movements, maneuvers and exercises. Scand. J. Rehabil. Med. (Suppl.), 1, 1-40.

15) Niu, C.C., Chen, W.J., Chen, L.H. \& Shih, C.H. (1996) Reduction-fixation spinal system in spondylolisthesis. Am. J. Orthop., 25, 418-424.

16) Panjabi, M.M., Krag, M.H. \& Goel, V.K. (1981) A technique for measurement and description of three-dimensional six degree-of-freedom motion of a body joint with an application to the human spine. J. Biomechanics, 14, 447-460.

17) Rohlmann, A., Riley, L.H., III, Bergmann, G. \& Graichen, F. (1996) In vitro load measurement using an instrumented spinal fixation device. Med. Eng. Phys., 18, 458488.

18) Rolander, S.D. (1966) Motion of the lumbar spine with special reference to the stabilizing effect of posterior fusion: An experimental study on autopsy specimens. Acta Orthop. Scand. (Suppl.), 90, 1-144.

19) Stovall, D.O., Jr., Goodrch, A., MacDonald, A. \& Blom, P. (1996) Pedicle screw instrumentation for unstable thoracolumbar fractures. J. South Orthop. Assoc., 5, $165-173$.

20) Suk, S.I., Lee, C.K., Kim, W.J., Lee, J.H., Cho, K.J. \& Kim, H.G. (1997) Adding posterior lumbar interbody fusion to pedicle screw fixation and posterolateral fusion after decompression in spondylolytic spondylolisthesis. Spine, 22, 210-220.

21) Weatherley, C.R., Prickett, C.F. \& O'Brien, J.P. (1986) Discogenic pain persisting despite solid posterior fusion. J. Bone Joint Surg. (Br.), 68, 142-143.

22) Weinhoffer, S.L., Guyer, R.D., Herbert, M. \& Griffith, S.L. (1995) Intradiscal pressure measurements above an instrumented fusion: A cadaveric study. Spine, 20, 526531. 